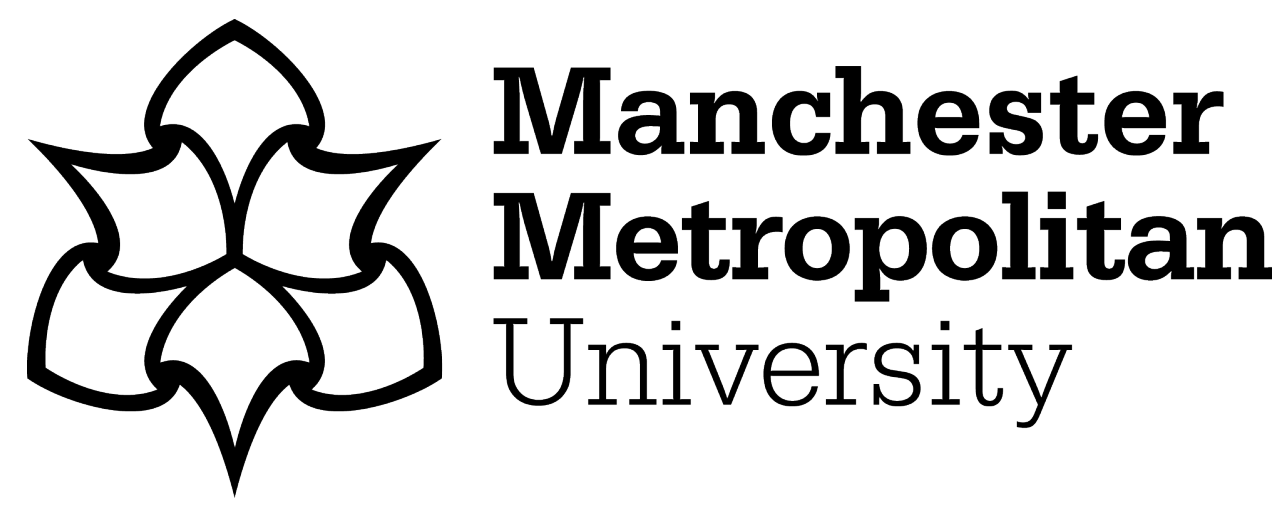

Boustila, Sabah ORCID logoORCID: https://orcid.org/0000-0001-6550913X, Capobianco, Antonio, Bechmann, Dominique and Genevaux, Olivier (2016) A Hybrid Projection to Widen the Vertical Field of View with Large Screens to Improve the Perception of Personal Space in Architectural Project Review. In: IEEE Symposium on 3D User Interfaces (3DUI), 19 March 2016 - 20 March 2016, Greenville, SC, USA.

Downloaded from: https://e-space.mmu.ac.uk/627768/

Version: Accepted Version

Publisher: IEEE

DOI: https://doi.org/10.1109/3DUI.2016.7460052

Please cite the published version 


\title{
A Hybrid Projection to Widen the Vertical Field of View with Large Screens to Improve the Perception of Personal Space in Architectural Project Review
}

\author{
Sabah Boustila* Antonio Capobianco ${ }^{\dagger} \quad$ Dominique Bechmann ${ }^{\ddagger} \quad$ Olivier Génevaux ${ }^{\S}$
}

University of Strasbourg, France

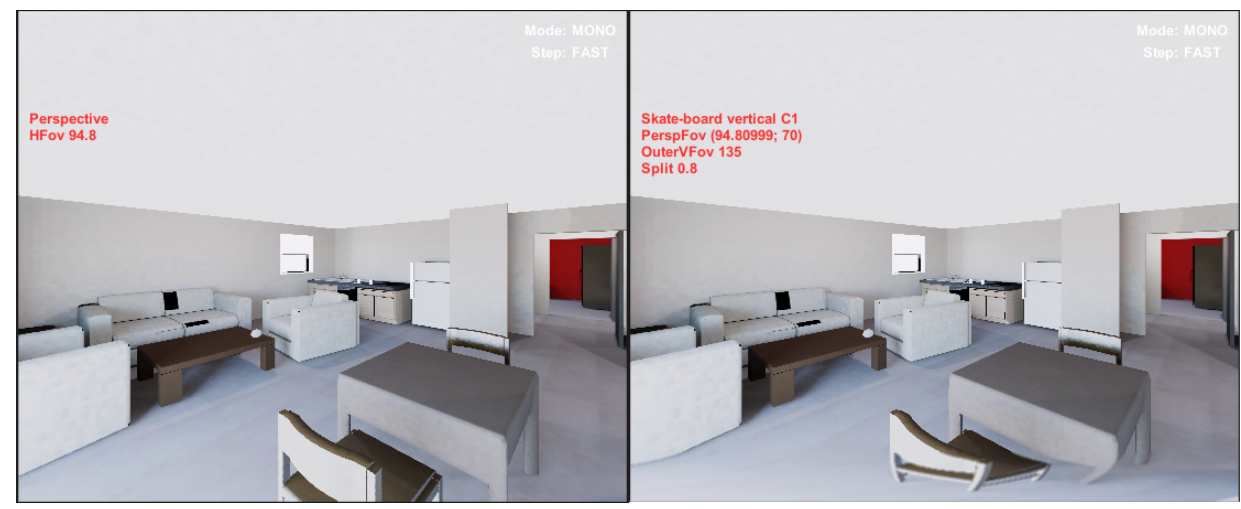

Figure 1: Left image represents a view with a perspective projection. Right image shows and example of the hybrid projection. In left image the user cannot see the chair behind him.

\begin{abstract}
In this paper, we suggest using an hybrid projection to increase the vertical geometric field of view without incurring large deformations to preserve distance perception and to allow the seeing of the surrounding ground. We have conducted an experiment in furnished and unfurnished houses to evaluate the perception of distances and the spatial comprehension. Results show that the hybrid projection improves the perception of surrounding ground which leads to an improvement in the spatial comprehension. Moreover, it preserves the perception of distances and sizes by providing a performance similar to the perspective one in the task of distance estimation.
\end{abstract}

Index Terms: H.5.1 [Information Interfaces and Presentation]: Multimedia Information Systems-Artificial, augmented, and virtual realities; I.3.7 [Computer Graphics]: Three-Dimensional Graphics and Realism - Virtual reality

\section{INTRODUCTION}

Due to the advances in computer technology, various virtual reality (VR) systems are available nowadays. Head Mounted Display (HMD), CAVE, curved screen, workbench and large screen are different technologies employed to immerse users in a synthetic world. However, the perception with these display devices differs from the

*e-mail: boustila@unistra.fr

†e-mail: a.capobianco@unistra.fr

†e-mail: bechmann@unistra.fr

§e-mail: e-mail: genevaux@unistra.fr perception in the real world in many aspects $[1,17]$. Prior studies found that distances are misperceived in virtual environments (VEs) $[6,7]$.

Human field of view (FoV) is different from the virtual one. The human has approximately a $200^{\circ}$ horizontal field of view (HFoV) and $135^{\circ}$ vertical field of view $(\mathrm{VFoV})$ with a binocular vision in motionless position [21]. Nevertheless, in VEs the FoV depends on the display device used. FoV is likely to be one of the factors influencing distance perception in VE and its impact has been investigated in several research studies $[1,10]$.

Our work is part of an architectural project aiming to integrate the VR tool in the process of architectural project review. The purpose of this use is to allow the prospects to review and validate during virtual visits, along with the architects, the mockups of houses in terms of: the size of the rooms, habitability which is the volume of the room and their quality to accommodate persons (capacity and comfort), and the layout of the rooms. During the virtual visits, the prospects and the architects take decisions based mainly on the perception of distances and volumes.

For this project, architects have chosen wall screen as display device because it allows 1) to immerse simultaneously the architects and the prospects in the scene to discuss of the plan of the house during its visualisation, 2) collaborative reviews at a lower cost in comparison to CAVEs. Our non-immersive wall screen used for this project offers $90^{\circ}$ of $\mathrm{HFoV}$ and $71^{\circ}$ of VFoV and does not provide projection on the ground (see figure 2 ). In this case, when the viewer stands at a distance of $1.5 \mathrm{~m}$ in front of the screen and sees the VE at first-person perspective view some parts of the personal space in the VE are not projected on the ground nor displayed on the wall screen. The personal space was defined as the zone immediately surrounding the observer's head up to $2 \mathrm{~m} \mathrm{[8]}$.

Though, the perception of the personal space seems to influence distance estimations in real environments [26, 23]. In the VE, 
Bruder et al. [5] reported that the distance between the observer and the screen of the CAVE and the parallax impact distance perception. Results have shown that estimations were different according to the distance from the screen and where the virtual object is displayed: in front of or behind the screen and at which distance.

In the context of virtual visits when using wall screens, the lack of the projection on the ground induces a problem of visibility of the personal space. Therefore, this problem may impact the perception of the area of the rooms, which is important for the understanding of their functionality and for assessing the habitability. Indeed, if the prospect visits small rooms such as the toilet or the bathroom (depth of the room less than $2 \mathrm{~m}$ ), if he/she cannot see the basic elements such as the toilet or the washstand, he/she cannot understand the role of this room and cannot understand the mockup of the house. However, this is very important for the project review. The problem of the visibility of the personal space also exists with large screen immersive displays (LSIDs) and 3D screens devoid of projection on the ground.

In this paper, we would like to resolve this problem by allowing the perception of the personal space on the wall screen. Our idea is to increase the vertical geometric FoV (VGFoV) to allow displaying nearby surrounding ground on the screen. We aimed to provide a VGFoV equivalent to the human $\mathrm{VFoV}\left(135^{\circ}\right)$.

Previous similar studies exist, though they studied the HFoV and not the VFoV [11, 2], these studies proposed to increase horizontal geometric FoV (HGFoV) based on rendering methods such as nonplanar projection. However, simply increasing the geometric FoV (GFoV) by regular projection distorts the VE. We thought to follow the same principle though, this is not suitable for our architectural context where the user needs a correct perception of the VE to judge distances and to understand the VE.

To alleviate such problem we followed the idea of $[16,20]$ dividing the screen in different zones of projection. In the present work, in the center of the screen $\left(71^{\circ}\right)$ the scene is rendered with a regular perspective projection while, in both top and bottom borders of the screen $\left(32^{\circ}\right)$ the scene is rendered with a non-planar projection (as illustrated in figure 3). Therefore, only the borders of the screen will be distorted.

With this study, we aimed to investigate whether increasing the VGFoV while keeping large areas without deformations allows the perception of the personal space without degrading the perception of distances and volumes. Our aim is not to improve the perception of distance but the perception of the personal space.

In this paper, we first present work that investigated the effect of FoV on the perception of distances in the VE, then we describe our hybrid projection. In the fourth section we explain our experiment to evaluate the effectiveness of the hybrid projection. The results are reported and discussed in section 5 and 6 respectively.

\section{Related WORK}

\subsection{Influence of FoV on perception}

One of the main factors which distinguishes the perception in real world and within VE is the FoV. Both horizontal and vertical FoV

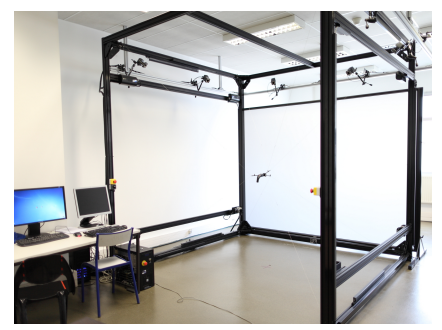

Figure 2: The large screen display device of our laboratory seem to affect differently the perception in the VEs [26]. Previous researches exploring FoV were focused on the HFoV rather than the VFoV.

There are several studies comparing distance perception in the VE among different display devices. Grechkin et al. [10] compared FoV with an HMD $\left(40.5^{\circ} \mathrm{X} 49.5^{\circ}\right.$ for horizontal and vertical FoV respectively), a LSID $\left(224^{\circ} \mathrm{X} 46^{\circ}\right)$ and real environment. Large differences in the HFoV produced similar underestimations with the two display devices for distances between 2 and $7 \mathrm{~m}$. Alexandrova et al. [1] conducted an experiment in real world and large semi-spherical immersive screen $\left(220^{\circ} \mathrm{X} 165^{\circ}\right)$. Distances were well estimated in the real environment while underestimated in the VE. Both results demonstrated that a wide HFoV does not lead to accurate estimations.

Changing the viewpoint in the real and VEs was also investigated in distance perception. Riecke et al. [19] kept the same FoV $\left(32^{\circ}\right.$ $\mathrm{X} 24^{\circ}$ ) for different display devices: HMD, 24" and 50" monitors and allowed changing the viewpoint in the VE. Estimations were accurate in all conditions. In contrast to this work, Corujeira and Oakley [6] compared distance perception with the same FoV $\left(32^{\circ}\right.$ $\mathrm{X} 24^{\circ}$ ) between HMD, computer screen and the real world but they did not allowed changing the viewpoint. Results have shown no difference between display devices. However, dissimilar to Riecke et al. [19] distances were underestimated in all conditions. In the real world, Creem-Regehr et al. [7] have reproduced a limited FoV $\left(42^{\circ} \mathrm{X} 32^{\circ}\right)$ similar to the one offered by an HMD. They examined restricted FoV combined with the possibility or not to change the viewpoint (head rotation) on egocentric distance judgements. Underestimation for distances between $4 \mathrm{~m}$ and $12 \mathrm{~m}$ was found only with limited HFoV without head rotation.

In our experiment, our large screen provides $90^{\circ}$ of $\mathrm{HFoV}$, participants were allowed to change the viewpoint to explore the VE. Thus, we avoid underestimations related to motionless in the VE.

In the work of Wu et al. [26] participants in motionless position looked at the real world through goggles. A first group experienced a restricted $\mathrm{VFoV}\left(50.9^{\circ}\right)$ and $29.2^{\circ}, 21.5^{\circ}, 14.3^{\circ} \mathrm{HFoV}$, while a second group experienced fixed $\mathrm{HFoV}$ of $57.7^{\circ}$ and $39.9^{\circ}, 29.6^{\circ}$ and $21.1^{\circ} \mathrm{VFoV}$. Distances were underestimated only with a VFoV restricted to $21^{\circ}$.

This last work suggests that the ground plane may contribute to the estimation of egocentric distances. Indeed, with $1.67 \mathrm{~m}$ eye height and $21^{\circ} \mathrm{VFoV}$, near surface ground up to $2.55 \mathrm{~m}$ was out of sight. The effect of seeing the near surface ground was also investigated in a second experiment in [26]. Results suggested that humans rely on near surface ground to evaluate distances. Besides, Sinai et al. [23] have found impairment of distance evaluation in real environment, when a target was placed behind a hole or on a surface ground with inhomogeneous texture.

\subsection{Existing techniques to widen the FoV}

Relevant studies that aimed to improve FoV suggested new devices that increase FoV. Simon and Gobel [22] proposed a device solution: the i-Cone. The device display is a screen with a conical shape. For rendering, they used a new projection-based panoramic display, with four projectors. This system enables a large HFoV of $240^{\circ}$. However, the VFoV remains limited because of the lack of projection on the floor.

Ardouin et al. [3] designed a novel display device called FlyVIZ. They used an HMD combined with an acquisition system, to provide real-time display of $360^{\circ} \mathrm{HFoV}$ and $80^{\circ} \mathrm{VFoV}$, without stereoscopic vision. The system processes omnidirectional images obtained from a panoramic sensor into an image of the surrounding environment. Other studies using panoramic camera with HMD exist, Onoe et al. [14], and Nagahara et al. [13] both propose an HMD that allows $180^{\circ} \mathrm{HFoV}$ and $60^{\circ} \mathrm{VFoV}$. The virtual view is driven by the viewing direction of the user, so he/she can explore a 
$360^{\circ} \mathrm{HFoV}$ just by changing the head orientation. Unlike, Orlosky et al. [15] proposed a prototype based on fisheye lenses to enlarge the peripheral vision with an HMD. They proposed two prototypes with $180^{\circ}$ and $238^{\circ}$ of HFoV. They used the Oculus Rift which initially provides $90^{\circ}$ of HFoV. To expand the HFoV, they kept $60^{\circ}$ undistorted central $\mathrm{FoV}$ for binocular vision and $30^{\circ}$ of peripheral vision. In these $30^{\circ}$, they compressed approximately $60^{\circ}$ of peripheral vision per eye. Thus, objects outside of the inherent viewing angle of the display become visible.

Otherwise, Tanaka et al. [24] constructed a system projection (TWISTER) allowing HFoV of $360^{\circ}$. The TWISTER is a new projection system formed by a few lines of vertical LEDs placed in cylinder support. This provides a cylinder environment where only one person can be inside. When the user is inside, the cylinder rotates to a pre-defined direction and the LEDs display a column of pixels. This principle provides an immersive autostereoscopic display without wearing 3D glasses. Unlike our aim, this system displays pictures and not 3D scenes so, the user cannot interact.

Other approaches that enhance the FoV proposed to increase the GFoV rather than the display FoV. Ardouin et al. [2] proposed a stereoscopic rendering with a wide $\mathrm{HGFoV}$ up to $360^{\circ}$. They mapped a $3 \mathrm{D} \mathrm{VE}$ to a $360^{\circ}$ display using a non-planar projection (equirectangular) and proposed a new pre-clip stage to handle the problem occurring with the polygons spanning across the projection discontinuities. The effectiveness of the rendering was evaluated using a monitor screen. However, understanding the VE becomes difficult with the great distortions introduced by the large HGFoV. Hu et al. [11] used the same principle of non-planar projection (cylindrical) to create a panoramic image from images captured by multiple digital cameras covering $360^{\circ}$ of the scene. The panoramic images were projected within an immersive environment in real-time.

In the context of Desktop VR, Robertson et la. [20] proposed a technique called Peripheral Lenses for simulating peripheral vision as a virtual navigation aid. The initial virtual scene is maintained in the center of the screen and the peripheral vision is mapped on panels on either side of the main display. Peripheral Lenses are viewpoints at the same origin as the main viewpoint, but oriented toward either side. Even if this technique allows peripheral vision, it does not improve the time of visual search task.

Furthermore, Petkov et al. [16] proposed a visualization technique for preserving shapes locally while increasing the GFoV up to $360^{\circ}$. The approach allows mapping between the full VE and an arbitrary display configuration such as CAVEs with 3,4 and 5 display surfaces and minimise the distortions in a large area in the middle of the screen.

In our context, the aim is close to the problems treated in the above mentioned work like widen the FoV and minimizing distortions. In our work, we provide an extension of the idea in [20, 15] to the VFoV rather than the HFoV.

\subsection{Our Approach}

With our display device where the user is located at $1.5 \mathrm{~m}$ away from the screen, a part of the personal space in the VE cannot be displayed. When participants visit rooms with small surface area in the house such as the toilet, the bathroom and even the kitchenette, if they cannot see the near surrounding surface ground and near objects they cannot understand the role of these rooms: toilet, bathroom, etc. (see left figure in figure 6). Therefore, at the end of the visit they cannot understand the layout of the rooms (where was the toilet, the bathroom, etc.) nor the mockup of the house. To address this problem we proposed to broaden the VGFoV, so that the user can see the personal space on the screen and better understand the VE.

In our work we suggest to increase the VGFoV while minimizing the distortions in the scene by using a hybrid projection. With this principle, our aim is not to improve the perception of distances and volumes but to not skew this perception of distances. We divide the display into three zones rendered with different ratios of GFoV to display FoV. For the rendering, we combine perspective and nonplanar projection (see section 3 ).

This work investigates whether keeping small zones of distortion 1) preserves the perception of distances and volumes and 2) improves the perception of the personal space. To evaluate the hybrid projection, we carry out virtual visits of houses to compare the perception of distances, volumes and the personal space between the hybrid and the perspective projections. Our hypothesis is that the hybrid projection improves the perception of the personal space and leads to better understand the VE.

Our contributions are:

1) A hybrid projection that increases the VGFoV while minimizing the distortions in the scene. With this method, we aim to avoid degrading the perception of distances and volumes.

2) A large VGFoV that improves the perception of the personal space missing because of the display device.

3) A rendering approach applicable for other display devices which not provide projection on the ground such as 3D screens.

\section{HYBRID PROJECTION}

\subsection{Purpose and description}

Given a non-immersive (monoscopic, with no head-tracking) walllike physical display device, our goal is to allow the user to see a large part of its surrounding virtual scene. Increasing the GFoV of a regular perspective projection cannot be done to a large extent unless too much distortions appear which hinders the perception of the VE. In this work, our interest is more specifically focused on expanding the VGFoV to provide feedback to the user about the virtual floor that lies close to him. This part of the scene is usually missing in such display device due to the distance between the user and the screen.

The solution we explore to address this goal is to display the scene using a combination of two different projection schemes. A regular perspective projection handles the main central part of the screen: it provides a distortion-free picture, albeit with limited VFoV. The remaining outer parts of the screen are handled by two cylindrical-like projections (one for each side) that enable a much larger VGFoV without incurring much distortions (as illustrated in the figure 3). This way, most of the image perceived by the user carries no distortion while a large part of the virtual scene can be perceived at the same time.

The screen area is divided in three zones as illustrated in the figure 3 , the choice of the angles was as follow:

- The total VGFoV is $135^{\circ}$. We chose $135^{\circ}$ to reproduce the human VFoV.

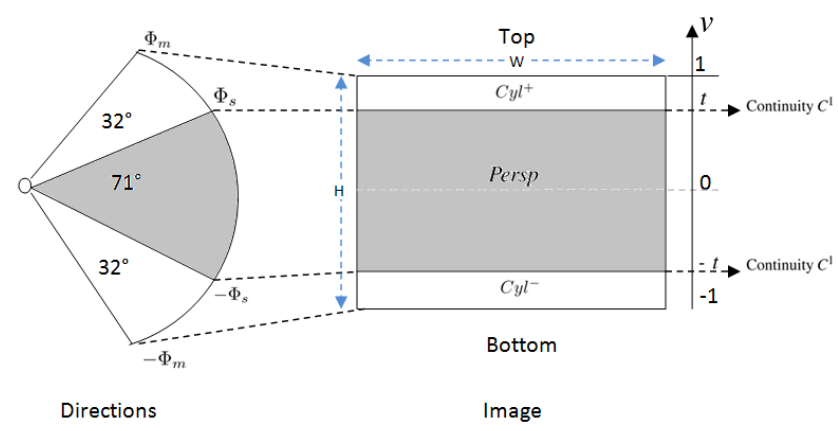

Figure 3: The principle of our hybrid projection and the different parameters used for the rendering. 
- In central area the GVFoV and the display VFoV are identical $\left(71^{\circ}\right)$. The scene is rendered with a perspective-like projection.

- In the top and the bottom areas, a larger VGFoV $\left(135^{\circ}-71^{\circ}\right.$ $=64^{\circ}$ for both strip) is mapped to the display VFoV with a cylindrical-like projection.

- The projection provides a symmetry relative to the horizontal and the vertical axis of the image.

\subsection{Technical details}

Central part of the image is handled by a regular perspective projection. Its parameters comprise the user-specified VGFoV for this screen section (in our experiment the perspective VGFoV was set to $71^{\circ}$ ) and the actual screen section aspect-ratio $\rho=w / h$. On each side of this central part, a cylindrical-like projection is used to widen the effective VFoV up to another user-supplied parameter. The effective parameters for these cylindrical-like projections are thus the outer VGFoV limits, in our experiment this parameter was set to $135^{\circ}$ and the screen-space geometry of the outer zones defined by the parameter $t \in I=[-1,1], I$ is the normalize image coordinates, as illustrated in figure 3 .

\subsubsection{Rendering}

Rendering a frame is done in two steps: pre-processing and image processing. First, in the pre-processing the scene is rendered offscreen into a highly-detailed cube-map centred around the user position in the virtual scene (i.e. a set of 6 textures mapped onto the faces a virtual cube surrounding the camera). This provides an easy way to access an image of the whole environment surrounding the user. Second, in the image processing, for each pixel $p$ of the final image, this cube-map is sampled along the proper viewing direction $(\theta, \Phi)$ depending on the position of the pixel $(u, v)$ on the screen, $(u, v) \in I=[-1,1]^{2}$.

This second step of image processing is performed by rendering a full-screen quad with an appropriate pixel shader. As the projection is not subjected to change, it is beneficial to precompute the viewing directions with respect to the user position for all pixels of the output image into a texture once, and to re-use these data every frame taking account of the current user position in the scene. This effectively reduces rendering of each frame to the update of the surrounding-environment cube-map and to a full-screen quad rendering featuring an indirect texture lookup whatever the actual projection scheme is.

\subsubsection{Details on the projections}

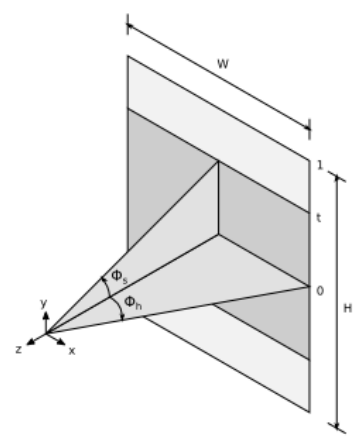

Figure 4: The space of directions used for the final rendering.

To compute the final pixel with shader based on our rendering technique, we used several parameters (see figures 3 and 4):
- $\Phi_{h}$ : the half horizontal aperture angle of the perspective projection.

- $\Phi_{s}$ : the half vertical aperture angle of the perspective projection.

- $\Phi_{m}$ : the half exterior vertical aperture angle of the cylindricallike projection.

- $t$ : the vertical position for the transition between the two projections ( $\mathrm{t} \in I=[-1,1], I$ is the normalize image coordinates)

The virtual viewpoint height was set to $112 \mathrm{~cm}$ corresponding to the origin of the projection angles. Here the key steps for the final rendering, from the cube map generated:

1) From a pixel on the final displayed image, we find the direction $(\theta, \Phi)$ to the space.

2) According to the location of the pixel on the three zones of the screen we choice the proper projection to calculate the direction $(\theta, \Phi)$. We applied whether the perspective projection $\Phi_{\text {persp }}(v)$ if the pixel is in the center of the screen or cylindrical-like projection if the pixel is in the top $\Phi_{c y l+}(v)$ or bottom $\Phi_{c y l-}(v)$ parts of the screen.

$$
\Phi(u, v)=\Phi(v) \begin{cases}\Phi_{c y l-}(v) & \text { if } v \leq-t \\ \Phi_{\text {persp }}(v) & \text { if }-t<v<t \\ \Phi_{c y l+}(v) & \text { if } v \geq t\end{cases}
$$

We compute the direction $(\theta, \Phi)$ from a pixel using the equations (2) for the perspective projection and the equations (3) for the cylindrical-like projection.

$$
\begin{aligned}
& \left\{\begin{array}{c}
\theta_{\text {persp }}(u, v)=\arctan \left(\frac{u \tan \Phi_{h}}{\sqrt{1+\left(\frac{v}{t} \tan \Phi_{s}\right)^{2}}}\right) \\
\Phi_{\text {persp }}(u, v)=\arctan \left(\frac{v}{t} \tan \Phi_{s}\right)
\end{array}\right. \\
& \left\{\begin{array}{c}
\theta_{c y l+,-}(u, v)=\theta_{\text {persp }}(u, t) \\
\Phi_{c y l+}(u, v)=\frac{v-t}{1-t}\left(\Phi_{m}-\Phi_{s}\right)+\Phi_{s} \\
\Phi_{c y l-}(u, v)=-\frac{v+t}{-1+t}\left(\Phi_{m}-\Phi_{s}\right)-\Phi_{s}
\end{array}\right.
\end{aligned}
$$

3) The pixels between the two projections parts are treated to keep smooth transition between parts, we imposed $C^{1}$ continuity between the central part and the outer parts using the constraints (4) for the top part and (5) for the bottom part.

$$
\begin{gathered}
\left\{\begin{array}{c}
\Phi_{\text {persp }}(u, t)=\Phi_{c y l+}(u, t)=\Phi_{s} \\
\frac{\partial \Phi_{\text {persp }}}{\partial v}(u, t)=\frac{\partial \Phi_{c y l+}}{\partial v}(u, t)
\end{array}\right. \\
\left\{\begin{array}{c}
\Phi_{\text {persp }}(u,-t)=\Phi_{c y l-}(u,-t)=-\Phi_{s} \\
\frac{\partial \Phi_{\text {persp }}}{\partial v}(u,-t)=\frac{\partial \Phi_{c y l-}}{\partial v}(u,-t)
\end{array}\right.
\end{gathered}
$$

For the outer parts of the image, the projection is not a regular cylindrical projection because we chose to enforce $C^{1}$ continuity across the jump in projection in order to hide the transition as much as possible. A regular cylindrical projection in which viewing angle linearly depends on screen-space position along each column of pixels is thus modified to accommodate for additional boundary conditions: prescription of endpoint values at each domain boundary (a),(c) and derivative value at inner endpoint (b), (d). We derive the appropriate quadratic polynomial to solve these constraints 
on each pixel column. As previously noted, computing the actual view direction for each pixel is not time-critical providing the results are cached and reused every frame: such computations can thus be done either using CPU or GPU.

\section{EXPERIMENTAL DESIGN AND MEASURES}

\subsection{Measures and evaluation}

To assess the effectiveness of the hybrid projection in the perception of distances and volumes and the spatial comprehension we have conducted virtual visits where the participant had to evaluate the size of the rooms, their furnishing and the habitability. Besides, he had to draw the plan of the house after each visit. Virtual visits were in furnished and unfurnished houses for investigating the influence of the presence of objects in the scene on the hybrid projection.

\subsubsection{Distance perception and habitability questionnaire}

This questionnaire comprises questions regarding the ability of the user to perceive and evaluate the distances, the sizes and the habitability. It features four categories of questions:

- Distance estimation: these questions required participants to estimate the size of the walls of the rooms: length and width using a metric system (meters), and the overall area of the house (in square meters). Since only few people can be accurate in expressing distances in meters or square meters, to keep answers within a reasonable estimations, the questions were closed-ended with 4 possible answers such as in [27]. These answers were calculated with an offset of (+/-) $15 \%$ of the veridical value. The offset was chosen so that it is higher than the just noticeable difference threshold for 3D distances [9] and allowing answers to remain in a reasonable interval. Participants were invited to consider the fourth answers and not just the responses of the middle (answer 2 and 3 ) to avoid systematic answers. Indeed, we have put the same number of questions with one, two and three overestimated answers for each questionnaire.

Example: choose from the following propositions the width of the wall: a) 4.2 , b) 4.9, c) $5.6, d) 6.3 \mathrm{~m}^{2}$.

- Size perception: participants were asked to evaluate the possibility (in term of space) to put furniture into the rooms at specific places. Questions were a 7-point Likert type scale questions.

Example: if you had to place the wardrobe along the wall on the side of the window, do you think that the space would be: 1) insufficient, 2), 3), 4), 5), 6), 7) sufficient.

- Habitability: it assesses the capacity of the space to accommodate persons and how their feeling of comfort would be. To do so participants were asked to evaluate their feeling of comfort and their capacity to project into the VE while imagining particular situations. Questions were a 7-point Likert type scale questions.

Example: When passing a person in the corridor, the space available would allow you to do it: 1) with difficulty,2), 3), 4), 5), 6), 7) easily.

For the two groups of questions size perception and habitability, the correct values were calculated with architects based on norms and rules of building construction in France. For example, in France for the habitability, minimal dimensions to respect are enforced, e.g. the minimum width of a corridor is $90 \mathrm{~cm}$. So that, a person needs a minimum width of $40 \mathrm{~cm}$, in order to cross another person in the corridor. We selected

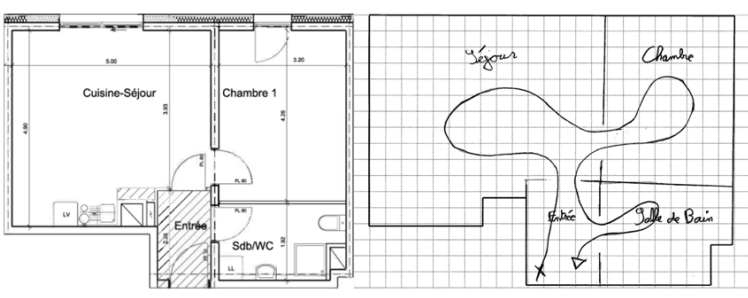

Figure 5: Example of SC task, left image illustrates a plan of a house, right image shows a cognitive map drawn by a participant.

questions which allow participants to respond with the right answer just by imagining the situation in question without the exact knowledge of these norms and rules.

Task difficulty: At the end of the visit, participants were asked to evaluate the level of the difficulty of the virtual visit and the estimation task by answering a 7-point Likert type scale question.

Example: Evaluate difficulty to visit and estimate the dimensions of the space: 1) difficult,2), 3), 4), 5), 6), 7) easy.

All questions were asked during the virtual visit, when the participants were seeing objects or rooms under inquiry.

\subsubsection{Spatial Comprehension (SC)}

Participants were asked to draw the plan of the house and the path of navigation after each visit, as illustrated in figure 5. This task evaluates the cognitive map created by the participants during the virtual visit. This allows us to evaluate if the hybrid projection improves the perception of the space and therefore improves the spatial comprehension. The plans were evaluated on 5 elements 1) the shape of the rooms, 2) the layout, 3) the size of the rooms and 4) their proportions, and 5) the order of the visit (the path of navigation). We attributed a subjective note on a scale of 5 ( 5 is the best note).

\subsubsection{Presence questionnaire (PQ)}

We evaluated presence with the standard Witmer and Singer questionnaire [25], a questionnaire of 19 Likert scale questions (scale from 1 to 7). We used this, to evaluate how the participant feels about his presence in the VE with the hybrid projection in comparison to the perspective projection.

\subsubsection{Debriefing}

At the end of the experiment, we gathered different information on: participant's metric knowledge (standard measure of furnitures and doors), the methodology used to answer questions, etc. during a semi-guided debriefing.

Example: 1) do you have knowledge on standard size of doors, beds, etc.

2) How did you elaborate your answers concerning: dimensions of rooms, size of furniture, etc.

\subsection{Method}

\subsubsection{Experiment design}

For the experiment, we used a between-group design. To evaluate the effectiveness of the hybrid projection method, we carried out virtual visits of houses, we compared the perception of distances and habitability and the spatial comprehension with both perspective and hybrid projections. During visits, participants had to navigate and to project inside the virtual houses and to evaluate distances and habitability through a questionnaire. To ensure that all subjects follow the same path and approximately get the same navigation time the visits followed a predefined path. The speed was 
fixed to a slow speed navigation $0.55 \mathrm{~m} / \mathrm{s}$ which is adapted to virtual visits in indoor scenes [4]. We tested two stimuli conditions: furnished houses and unfurnished houses to investigate the influence of the furniture on the hybrid projection. We compared results between hybrid and perspective projections. The four experimental conditions are:

(1) Furnished houses + perspective projection;

(2) Unfurnished houses + perspective projection;

(3) Furnished houses + hybrid projection;

(4) Unfurnished houses + hybrid projection.

\subsubsection{Participants}

Forty individuals participated in the experiment, aged between 19 and 32 with an average of about 24 . Ten individuals were assigned to each conditions. Participants were students in the computer graphic field in our university and were all voluntary. All of them reported normal or corrected-to-normal vision.

\subsubsection{Stimuli and apparatus}

The virtual visits took place in two kind of houses: furnished and unfurnished. For the first type, houses were furnished with sofa, tables, chairs, beds, bedside tables, wardrobes. For the second type, rooms were empty and only basic elements were present such as a toilet, shower, washbasin and doors. Virtual houses were reproduced from real floorplans. All houses were at scale 1:1 and the size of the walls was between 2 and $7 \mathrm{~m}$. Houses were modeled using 3DMax software, with simple textures. Realistic lighting was precomputed to avoid latency.

This experiment took place within the virtual reality platform In Virtuo of our laboratory. The VE was displayed on large screen (3 $\mathrm{m} \mathrm{X} 2.25 \mathrm{~m}$ ), with monoscopic vision and without head-tracking. For the navigation, we used the Ninento Wiimote as interaction device. The rendering allows only horizontal rotation (rotate around oneself), rotations up and down were not provided. Participants sit on an adjustable chair height $1.5 \mathrm{~m}$ away in front of the screen with eye-height fixed to $112 \mathrm{~cm}$. Riecke et al. [19] in their experiment have used an eye-height of $110 \mathrm{~cm}$, distance estimations were accurate with non-immersive large screen. The VE was rendered using the Unity 3D game engine.

\subsubsection{Procedure}

We compared the perception of distances and habitability between four conditions as presented in section 3.2.1. In each condition participants visit 4 different houses. The duration of the virtual visits is about 35 minutes for the four houses and the total duration of the experiment is 50 minutes including visits and questions.

Pre-experiment. Prior to the experiment, the participant reads instructions which explain the progress of the experiment, the technique of the navigation and the questionnaires to answer. Then, the participant trains on the navigation technique in the VE during four minutes. Before starting the experiment, the experimenter shows a measuring tape, with exactly 1 meter to the participant for few seconds to ensure that all participants have the same minimum metric knowledge to answer questions.

Experiment. During the experiment, the participant visits 4 houses. In each tour he visits all the rooms, starting by the entrance of the house. Then passing through the living room, kitchen, rooms then bathroom and toilet. In the experiment the visit is done with guided navigation following a predefined path represented by a green breadcrumb trail in the scene, to ensure that all participants have the same visit and the same time in the VE. For the navigation, the participant uses buttons on the wiimote, he has only the possibility to move forward, to stop, and to turn around himself, rotations up and down were not allowed nor provided by the system.

When the participant visits a room, he hears a beep at predefined stop points, he gets stuck with only the ability to look around

\begin{tabular}{|c|c|c|c|c|c|c|}
\hline Projection & $\begin{array}{c}\text { Distance } \\
\text { estimation }\end{array}$ & $\begin{array}{c}\text { Size } \\
\text { perception }\end{array}$ & Habitability & $\begin{array}{c}\text { Task } \\
\text { difficulty }\end{array}$ & SC & PQ \\
\hline HP & 0.484 & -1.039 & -0.693 & 4.222 & 3.934 & 4.633 \\
PP & 0.360 & -0.121 & -0.157 & 4.369 & 3.692 & 4.419 \\
\hline $\mathrm{F}(1,36)$ & 0.026 & 0.023 & 0.002 & 1.584 & 9.103 & 3.554 \\
p & 0.874 & 0.881 & 0.967 & 0.225 & 0.007 & 0.07 \\
\hline
\end{tabular}

Table 1: All mean, $F$ and $p$ values showing the influence of the type of projection on the dependent variables and SC and PQ.

\begin{tabular}{|c|c|c|c|c|c|c|}
\hline $\begin{array}{c}\text { Furnishing } \\
\text { condition }\end{array}$ & $\begin{array}{c}\text { Distance } \\
\text { estimation }\end{array}$ & $\begin{array}{c}\text { Size } \\
\text { perception }\end{array}$ & Habitability & $\begin{array}{c}\text { Task } \\
\text { difficulty }\end{array}$ & SC & PQ \\
\hline FUR & 0.351 & 0.925 & -0.529 & 4.065 & 4.345 & 4.567 \\
UNFUR & 0.444 & -0.445 & -0.503 & 4.5 & 3.270 & 4.479 \\
\hline F(1,36) & 0.037 & 0.030 & 0.030 & 2.127 & 18.982 & 8.218 \\
p & 0.850 & 0.865 & 0.863 & 0.163 & $<0.001$ & 0.010 \\
\hline
\end{tabular}

Table 2: All mean, $F$ and $p$ values showing the influence of the furnishing condition (FUR: furnished, UNFUR: unfurnished) on the dependent variables and SC and PQ.

himself. At this moment, the experimenter starts asking the questions regarding distance perception and habitability concerning the current room and notes the answers. Afterwards, the experimenter release the participant to visit the next room. After each visit, the participant carries out the SC, he draws the mockup of the house and the navigation path.

Post-experiment. The participant answers the PQ at the end of the experiment and gives a debriefing.

\section{RESULTS}

To apply a parametric test in the analysis we used the mean values of question categories (distance perception, size perception and the habitability) which contain several questions. For the statistical analysis, we first performed the test of Shapiro-Wilk and Bartlett to verify if our data fulfilled the conditions of normality and homogeneity. Since these conditions are respected, we realised multifactorial ANOVA at the 5\% significance level. Our independent variables are the projection (hybrid and perspective) and the furnishing condition (furnished and unfurnished). The dependent variables are: distance estimation, size perception, habitability and task difficulty. We also analysed the effects of the projection on the spatial comprehension (SC) and the presence (PQ).

When the multi-factorial ANOVA showed a significant influence of the independent variables we ran a pairwise comparison using the Tukey HSD (Honestly Significant Differences) test.

\subsection{Distance estimation}

For the analysis of distance estimation, we calculated the gap with the veridical distance. If it is null, the estimation is proper. Otherwise, if it is positive, the evaluation is overestimated, and underestimated, if it is negative. For statistical reasons, in our questionnaire we have put the same number of questions where the veridical distance was in the first, second, third and fourth answer.

In all conditions, distances were slightly overestimated. As illustrated in the boxplot (a) in figure 7, estimations were distributed above zero, with almost $50 \%$ of values between 0 and 1 which means that distances were overestimated within a maximum of $15 \%$ of the veridical distance.

Both of independent variables: projection (table1: $\mathrm{F}(1,36)=$ $0.026, \mathrm{p}=0.874$ ) and furnishing condition (table 2 : $\mathrm{F}(1,36)=0.037$, $\mathrm{p}=0.850$ ) had no significant main effect on distance estimation. 


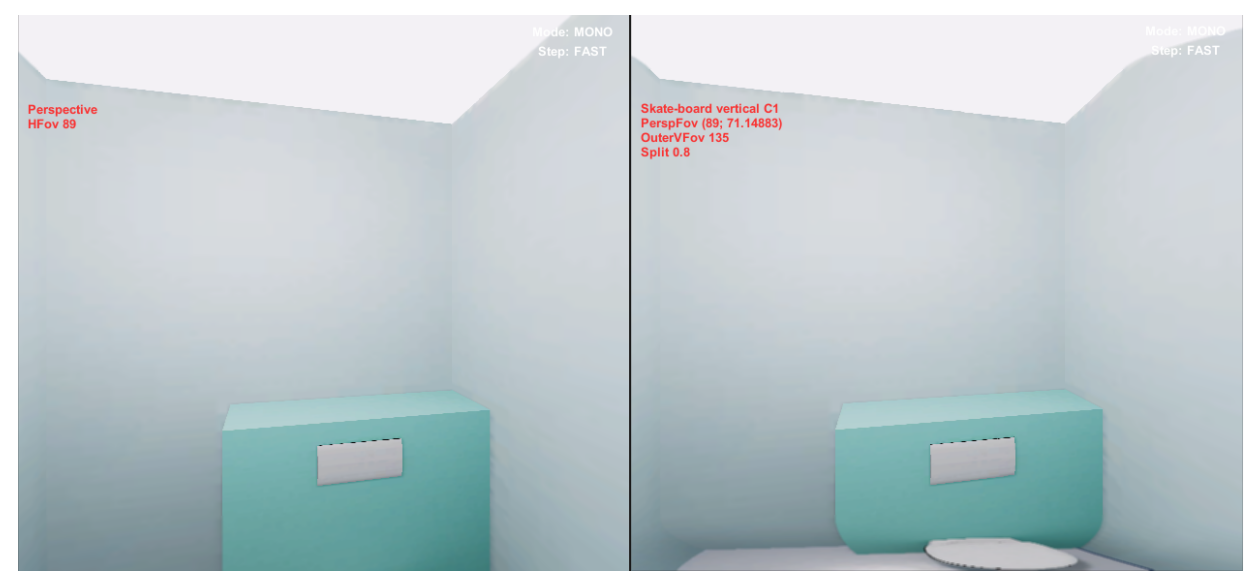

Figure 6: Left and right figures show the view of the scene that see the participant when he enter to the toilet with the perspective and the hybrid projection respectively. In right figure the participant can better understand the functionality of the room when seeing the toilet seat.

\subsection{Size perception and habitability}

In the analysis of results, we compute the signed distance between the correct answer and the participant's answer. If it is null, the estimation is exact. If it is positive, so the evaluation is overestimated, otherwise, the evaluation is underestimated.

In contrast to distance estimation, size perception and habitability overall values show a tendency of the subjects to underestimate sizes and the habitability of the houses (for the distribution of values see boxplots (b) and (c) in figure 7). The analysis have shown no main effect of the projection (table $1: \mathrm{F}(1,36)=0.023, \mathrm{p}=0.881$ ) and furnishing condition (table $2: \mathrm{F}(1,36)=0.030, \mathrm{p}=0.0 .865$ ) on size perception.

Alike, no significant main effect was found for the projection (see table 1: $\mathrm{F}(1,36)=0.002, \mathrm{p}=0.967$ ) and the furnishing condition (see table 2: $\mathrm{F}(1,36)=0.030, \mathrm{p}=0.863$ ) on habitability. However, we found a significant interaction effect between the furnishing condition and the projection $(\mathrm{F}(1,36)=4.674, \mathrm{p}=0.045)$ on habitability, as illustrated in figure 8 . This interaction suggest that we should ignore the absence of main effect of the factors, and investigate the simple main effects of our independent variables separately. Thus, we divided our dataset along each level of our projection variable and ran an ANOVA investigating the impact of furnishing condition on habitability (at a .025 level of significance, to take into account the multiple ANOVA). We found that the furnishing condition has a significant impact on habitability for the perspective projection $(\mathrm{F}(1,18)=7.378, \mathrm{p}=0.0142)$ but not for the hybrid projection $(\mathrm{F}(1,18)=0.361, \mathrm{p}=0.556)$. For the perspective projection, the habitability is overestimated in the unfurnished condition, and underestimated in the furnished condition. No such phenomenon is observed for the hybrid projection, the habitability being underestimated in both cases, however more accurate than with perspective projection (see figure 8).

\subsection{Spatial comprehension (SC)}

The spatial comprehension was evaluated with a subjective note on the scale of 5 , with 5 the best note. Values reported represent a mean note on 5 . The analysis have shown that both of the independent variables, projection (see table $1: \mathrm{F}(1,36)=9.103, \mathrm{p}=0.007$ ) and furnishing condition (see table $2: \mathrm{F}(1,36)=18.982, \mathrm{p}<0.001$ ) had a significant effect on the SC.

For the projection, participants had better results in the hybrid projection (mean note of 3.934/5) than in the perspective one (mean note of 3.692/5), see table 1 .

Concerning the furnishing condition, in furnished houses participants had a good score with a mean score of $4.345 / 5$ while in unfurnished houses participants had a less score with a mean score of $3.270 / 5$, see table 2 .

\subsection{Task difficulty}

No significant influence of the projection (see table 1: $F(1,36)=$ $1.584, \mathrm{p}=0.225$ ) and furnishing condition (see table $2: \mathrm{F}(1,36)=$ $2.127, p=0.163$ ) was found on task difficulty. On average, the task difficulty was estimated as being relatively high for both projections, with an overall mean values of 4.222 and 4.369 (for hybrid and perspective projection respectively) on a scale of 7 ( 7 is being difficult).

\subsection{Presence Questionnaire (PQ)}

The presence was a note on the scale of 7 ( 7 is well immersed). We found a tendency of the projection on the presence (table 1: $\mathrm{F}(1,36)$ $=3.554, \mathrm{p}=0.076$ ). Participants with the hybrid projection had a tendency to feel more present in comparison to participants with the perspective projection.

As for the furnishing condition, a significant influence was found on the presence $(F(1,36)=0.218, p=0.010$, see table 2$)$. Participants in furnished houses felt more present than those in unfurnished houses.

\section{Discussion}

\subsection{Distance perception and habitability questionnaire}

Results have shown that distance estimation was overestimated, whereas size perception and habitability were underestimated. These results indicate that the estimation of distances and volumes is different with respect to the category of questions. We can interpret these results by the effect of the method used to give estimations. Several previous studies have found that the estimation of distances and the accuracy are dependent on the applied measurement method. Notably, how participants express the perceived distance $[18,10]$. Indeed, in the experiment, the way to interpret the perceived distance was different according to the category of questions. In distance estimation, the evaluation was with a metric measure (meter or meter square), for this, participants chose one response among four propositions. Though, size perception and habitability were questions on a Likert scale, so the participant has to imagine situations and based on the perceived distance he gave the answer. This difference in the manner to interpret the perceived distance creates the difference in accuracy between the categories of questions. Same results were found in the previous study in Boustila et al. [4], distances were overestimated when evaluated 

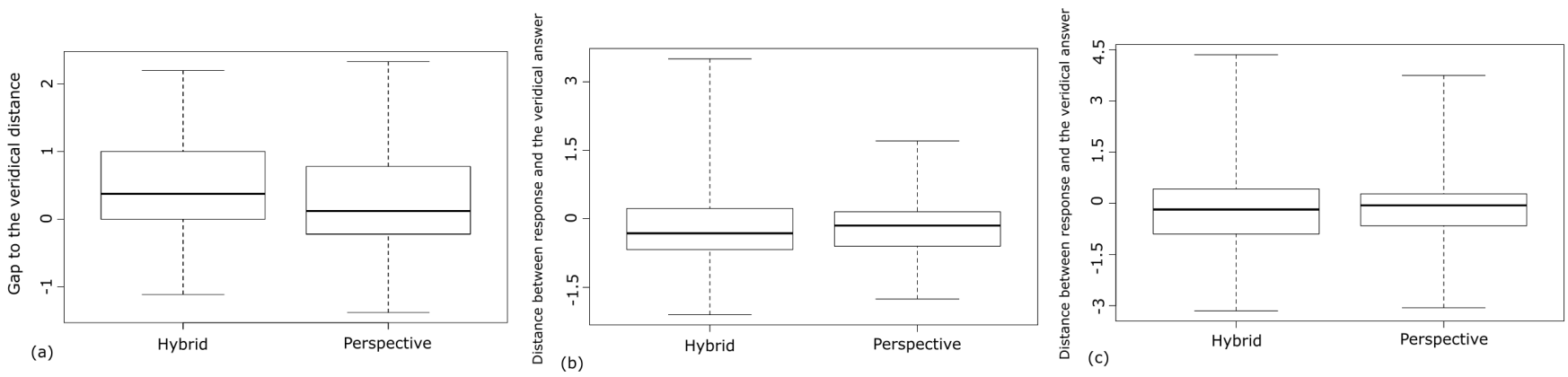

Figure 7: (a), (b) and (c) Plots of the results of distance estimation, size perception and habitability per projection: hybrid and perspective, respectively.

with a metric measures while size perception and habitability were underestimated when evaluated based on imagining situations.

Moreover, the strategy used to give answers can be the origin of this difference between questions: distance estimation and both size perception and habitability. Indeed, during the experiment, the strategy used to give answers by participants was different between a quantification (distance estimation) and a projection in the VE (size perception, habitability). This is confirmed by the reports during the debriefing. Participants mentioned using a computational strategy to calculate the veridical distance for distance estimation. They calculated the size of the wall by imagining a segment of one meter, or a segment equivalent to their height along the wall. These computational strategy can be the reason of the overestimation. Indeed, if the participants imagine a segment less than one meter (or their height) and consider it as one meter (or equal to their height) they will put more segment than necessary which leads to an overestimation. However, for size perception and habitability the strategy was not the same, participants tried to project into the environment and to imagine the situations requested to give an answer.

Further, the overestimation in distance estimation questions is consistent with the result of Zikic [27]. In this study, an overestimation occurred when the participants estimated the dimensions of the rooms in houses with wall screen. Estimations were indicated in feet.

As shown in the results, the hybrid projection provides the same performance than the perspective one in the perception of distances and habitability (distance estimation, size perception and habitability). These results, confirm that the hybrid projection preserve the perception of distances and volumes. Therefore, as we expected, the hybrid projection allows to 1) increase the VGFoV and 2) preserve the perception of distances and volumes. With this rendering technique, distances were preserved in a large part of the screen which led to the same performance between the two projections. This idea is confirmed by the results of the debriefing. Participants reported relying on the center of the screen rather than the outer parts to answer the questions.

\subsection{Spatial comprehension}

Results indicated that participants with hybrid projection had a better score in SC than participants with the perspective one. This means that participants with the hybrid projection understand better the plan of the house during the virtual visit, so that at the end of the visit they were able to create a best cognitive map in comparison to the participants with the perspective projection. This difference can be related to the problem of the perception of the personal space present with the perspective projection. Indeed, with the regular perspective projection ( $\mathrm{VFoV}$ of $71^{\circ}$ ) in small spaces such as the toilet and the bathroom where the depth of the rooms is less than
2 meters (as illustrated in figure 6), the participants at the entrance of the room cannot see or see just a part of the basic elements such as the toilet seat, the washbasin and bathtub, when they are situated at a distance less than $1.5 \mathrm{~m}$ from them (see left figure in figure 6). Therefore, they did not understand the role of these rooms and they were unable to understand the layout of the house at the end of the visit (which room was next to the other). Thus, they were unable to create an accurate cognitive map. Moreover, during the debriefing session some participants have indicated visiting small rooms but they were unable to remember the significant and the layout of these rooms. Other participants indicated forgetting the place of the toilet or the bathroom in the house and even doubt that they were in the house.

However, with the hybrid projection when the participants visit these rooms, they can see the basic elements such as the toilet seat, as illustrated in the right figure in figure 6. Therefore, they had a complete image of the room and its role, so at the end of the visit they create an accurate cognitive map. These results confirm that the hybrid projection improves the perception of the personal space and leads to better understand the VE.

Otherwise, results have shown that participants in furnished houses had a better score in SC than participants in unfurnished houses, this is consistent with the previous result in [4]. In fact, the presence of the furniture in the rooms helps participants to better understand the role of the rooms (living room, bed room, etc.) and allows them to remember the environment after the virtual visit. However, in unfurnished houses, even if the experimenter provides indications about the functionality of the rooms through the questions, the indications seem to not help them, e.g. if you had to fit out the room as a bedroom with a large double bed and two bedside tables near the wall of the window, do you think that the space would be sufficient?. These allowed them to understand the role of the rooms during the visit but it was insufficient to allow them remembering the rooms after the visit. This result confirms previous finding that the presence of the furniture improves the spatial comprehension [4].

\subsection{Furnishing conditions}

Results have shown that the furnishing condition had no significant influence on the perception of distances and habitability and task difficulty. During the debriefing, participants reported using a computational strategy based on participants height or the segment of one meter shown before the visit, and not on the size of the furnitures present in the scene to estimate distances and habitability, even for furnished houses. This computational strategy may explain why the furnishing condition had not significant effects.

As for the projection, we found a significant interaction between the furnishing condition and the projection on the evaluation of the 
habitability. This interaction shown that when investigating the furnishing condition with the two projections separately, we found a main effect of the furnishing condition on the habitability with the perspective projection. In this case, the habitability was overestimated in unfurnished houses and underestimated in furnished houses. However, no significant effect was found on the habitability with the hybrid projection, participants estimated the habitability (with an underestimation) with the same performance in both furnished and unfurnished houses.

One possible reason for this difference can be the perception of the scale. To evaluate the habitability e.g. if you wont to organize a party in this room and invite 10 persons, do you think that the space would allow you to feel: 1) uncomfortable, ...,7) comfortable, the participants (in furnished and unfurnished houses) during debriefing have indicated that they tried to imagine persons in the room and to evaluate if the space can accommodate the number of persons in the question. Maybe in the furnished houses, in both projections, participants underestimate the space between furniture and therefore underestimate the number of persons and the habitability. In figure 8 , the habitability is more accurate with hybrid projection than with the perspective one, but steel not significant.

In the unfurnished houses, participants used the same strategy to evaluate the habitability however, they had to imagine also furniture. Thus, maybe when imagining the furniture they imagine with an approximative scale and not with the real scale (small furniture). Therefore, they overestimate the possible number of persons in the room and overestimate the habitability with the perspective projection. Unlike in the hybrid projection, the participants misperceive the scale of the deformed parts so, when evaluating the habitability on these parts they underestimate the space which leads to underestimate the habitability. It is important to underline that this difference between projections is not significant as mentioned in the results, thus our conclusion that the hybrid projection preserve the perception of the habitability steel valid.

The same performance with the hybrid projection when estimating the habitability with furnished and unfurnished houses can confirm our interpretation of the perception of the scale. Indeed, the presence of furniture in furnished houses allows participants to perceive the scale on the deformed parts and to evaluate the habitability with the same performance than participants with the perspective projection. However, the absence of the furniture in unfurnished houses prevents the participants to perceive the scale of the deformed parts and led to underestimate the habitability, while it was overestimated with the perspective projection. The problem of the perception of the scale has been investigated in previous work [12], which suggested that the presence of some cues of familiar size can specify absolute distances.

We conclude that in the hybrid projection, the presence of objects in the VE improves the perception of the scale in the deformed parts

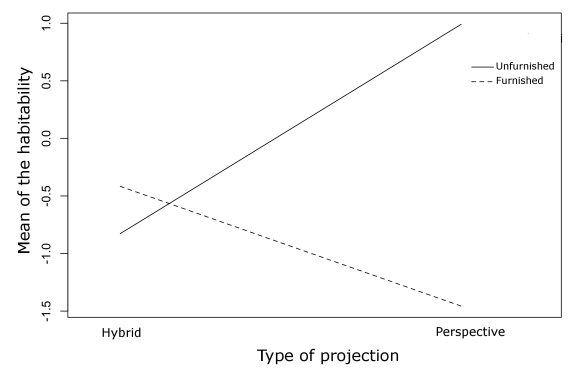

Figure 8: Interaction between habitability, projection and furnishing condition. and helps in the perception of distances and volumes. Therefore, we recommend to use the hybrid projection with furnished houses in the context of virtual visits. This suggestion is consistent with guidelines proposed in [4] for architectural project review.

\subsection{Task difficulty and presence}

The hybrid projection was also suitable for virtual visits. This is shown by the result of task difficulty, where participants had reported same level of difficulty in both projections. The task of the navigation and the estimation of distances and habitability was not more complicated even with the presence of the deformed zones (cylindrical projection).

Furthermore, the hybrid projection seems to provide a good sense of presence. Result has shown that participants using the hybrid projection had a tendency to feel more present compared to participants in the perspective one. In fact, during the debriefing session, the participants with the hybrid projection mentioned that when navigating, the deformations on the outer parts of the screen have encouraged them to stay focused on the screen and on the virtual visit. Thus, they had a good sense of presence. Otherwise, this effect could be related to the VGFoV of $135^{\circ}$ which corresponds to the natural human one. The large $\mathrm{VFoV}$ of the hybrid projection probably provides a close to natural perception which improves the sense of presence in comparison to the perspective projection.

\subsection{Lack of the projection}

In the hybrid projection, we fixed the user's head position to precompute the cube-map for the rendering. However, it is possible to use the head-tracking with this projection. To do so, the cube-map must be recumputed according to the user's head position whenever the user changes his head position. Moreover, in our experiment we have used monocular vision, the stereoscopy can be added in the future. In that case, the calculations of the projection must be done twice, one per eye tacking into account the interocular distance.

\section{CONCLUSION AND FUTURE WORK}

Architectural project review in virtual environment allows the evaluation of the digital mockups through virtual visits at real scale. Architects and prospects assess the size of the rooms and the habitability (the comfort and capacity of the rooms to accommodate persons), afterwards they take decisions. Wall screen display devices provide a collaborative environment at reasonable cost compared to CAVEs. However, when the user stands in front of the screen at some distance (d) and visits the house at first-person perspective view, a part of the scene is missing owing to the lack of the projection on the ground. In fact only objects at distance greater than (d) are displayed on the screen. This problem can affect the virtual visits in the VE. To resolve this problem we proposed to widen the vertical geometric field of view (VGFoV) to allow the observing of the personal space on the screen.

In this paper, we use a hybrid projection which provides a large $\mathrm{VGFoV}$ of $135^{\circ}$, the horizontal field of view being of $90^{\circ}$. In this technique we combine two kinds of projections. In the center of the screen, a $71^{\circ}$ of the VGFoV is rendered with a perspective projection while, the upper and lower parts of the screen (a $32^{\circ}$ of VGFoV for each part) are rendered with a like-cylindrical projection. The aim of this rendering technique is to improve the perception of the personal space without degrading the perception of distances and habitability. We have conducted an experiment in furnished and unfurnished houses to compare the perception of the personal space, the distances and the habitability between the hybrid projection and the perspective one. Results reveal that the hybrid projection provides same performance in the perception of distances and volumes than the perspective one. Furthermore, it improves the perception of the personal space by an improvement of the perception of small rooms (toilet, bathroom) and leads to better understand the VE. It 
also gives a better sense of presence. Otherwise, we suggest to use the hybrid projection with virtual scenes that contain objects providing information about the scale, to better understand the scale of the deformed parts. For future improvements, the projection can be used with the stereoscopic vision and the head tracking.

With the perspective projection, when navigating in the virtual environment the user can bump into nearby objects that are not displayed on the screen. In future work we suggest to investigate the influence of the hybrid projection on the navigation task.

\section{ACKNOWLEDGEMENTS}

The authors would like to thank Frédéric Larue, Sylvain Thery and Alexandre Hurstel for assistance with the implementation. This work was supported by the project CIMBEES FUI FEDER.

\section{REFERENCES}

[1] I. V. Alexandrova, P. T. Teneva, S. de la Rosa, U. Kloos, H. H. Bülthoff, and B. J. Mohler. Egocentric distance judgments in a large screen display immersive virtual environment. In Proceedings of the 7th Symposium on Applied Perception in Graphics and Visualization, APGV'10, pages 57-60. ACM, 2010.

[2] J. Ardouin, A. Lécuyer, M. Marchal, and E. Marchand. Stereoscopic rendering of virtual environments with wide field-of-views up to $360^{\circ}$. In Virtual Reality (VR), 2014 IEEE, pages 3-8, 2014.

[3] J. Ardouin, A. Lécuyer, M. Marchal, C. Riant, and E. Marchand. FlyVIZ: A novel display device to provide humans with $360^{\circ}$ vision by coupling catadioptric camera with hmd. In Proceedings of the 18th ACM Symposium on Virtual Reality Software and Technology, pages 41-44. ACM, 2012.

[4] S. Boustila, A. Capobianco, and D. Bechmann. Evaluation of factors affecting distance perception in architectural project review in immersive virtual environments. In Proceedings of the 21st ACM Symposium on Virtual Reality Software and Technology, VRST '15. ACM, 2015.

[5] G. Bruder, F. Argelaguet Sanz, A.-H. Olivier, and A. Lécuyer. Distance Estimation in Large Immersive Projection Systems, Revisited. In IEEE Virtual Reality, Arles, France, Mar. 2015.

[6] J. G. P. Corujeira and I. Oakley. Stereoscopic egocentric distance perception: The impact of eye height and display devices. In Proceedings of the ACM Symposium on Applied Perception, pages 23-30. ACM, 2013.

[7] S. H. Creem-Regehr, P. Willemsn, A. A. Gooch, and W. B. Thompson. The Influence of Restricted Viewing Conditions and Egocentric Distance Perception: Implications for Real and Virtual environments. Perception, 34:191-204, 2005.

[8] J. E. Cutting and P. M. Vishton. Chapter 3 perceiving layout and knowing distances: The integration, relative potency, and contextual use of different information about depth*. In W. Epstein and S. Rogers, editors, Perception of Space and Motion, Handbook of Perception and Cognition, pages 69 - 117. Academic Press, San Diego, 1995.

[9] D. De Silva, W. Fernando, S. Worrall, S. Yasakethu, and A. Kondoz. Just noticeable difference in depth model for stereoscopic $3 \mathrm{~d}$ displays. In Multimedia and Expo (ICME), 2010 IEEE International Conference on, pages 1219-1224, July 2010.

[10] T. Y. Grechkin, T. D. Nguyen, J. M. Plumert, J. F. Cremer, and J. K. Kearney. How Does Presentation Method and Measurement Protocol Affect Distance Estimation in Real and Virtual Environments? ACM Trans. Appl. Percept., 7(4):26:1-26:18, 2010.

[11] O. Hu, C. Kurashima, and R. Lopes. Panoramic images composition with positioning maps for virtual telepresence. In IX Symposium on Virtual and Augmented Reality SVR 2007, 2007.

[12] J. Loomis and J. Knapp. Visual perception of egocentric distance in real and virtual environments. Virtual and Adaptive Environments: Applications, Implications, and Human Performance Issues, 2003.

[13] H. Nagahara, Y. Yagi, and M. Yachida. Wide field of view head mounted display for tele-presence with an omnidirectional image sensor. In Computer Vision and Pattern Recognition Workshop, 2003., volume 7, pages 86-86, 2003.
[14] Y. Onoe, N. Yokoya, K. Yamazawa, and H. Takemura. Visual surveillance and monitoring system using an omnidirectional video camera. In ”Pattern Recognition, 1998. Proceedings. Fourteenth International Conference on, volume 1, pages 588-592 vol.1, 1998.

[15] J. Orlosky, Q. Wu, K. Kiyokawa, H. Takemura, and C. Nitschke. Fisheye vision: Peripheral spatial compression for improved field of view in head mounted displays. In Proceedings of the 2Nd ACM Symposium on Spatial User Interaction, SUI '14, pages 54-61, New York, NY, USA, 2014. ACM.

[16] K. Petkov, C. Papadopoulos, M. Zhang, A. E. Kaufman, and X. Gu. Interactive visibility retargeting in $\mathrm{vr}$ using conformal visualization. IEEE Transactions on Visualization and Computer Graphics, 18(7):1027-1040, jan 2012.

[17] R. S. Renner, B. M. Velichkovsky, and J. R. Helmert. The perception of egocentric distances in virtual environments - a review. ACM Comput. Surv., 46(2):23:1-23:40, 2013.

[18] A. R. Richardson and D. Waller. Interaction with an immersive virtual environment corrects users' distance estimates. Human Factors, 49(3):507-517, 2007.

[19] B. E. Riecke, P. A. Behbahani, and C. D. Shaw. Display size does not affect egocentric distance perception of naturalistic stimuli. In Proceedings of the 6th Symposium on Applied Perception in Graphics and Visualization, pages 15-18. ACM, 2009.

[20] G. Robertson, M. Czerwinski, and M. van Dantzich. Immersion in desktop virtual reality. In Proceedings of the 10th Annual ACM Symposium on User Interface Software and Technology, UIST '97, pages 11-19, New York, NY, USA, 1997. ACM.

[21] L. D. Silverstein. Foundations of vision. Color Research and Application, 21(2):142-144, 1996.

[22] A. Simon and M. Gobel. The i-cone trade; - a panoramic display system for virtual environments. In Computer Graphics and Applications, 2002. Proceedings. 10th Pacific Conference on, pages 3-7, 2002.

[23] M. J. Sinai, T. L. Ooi, and Z. J. He. Terrain influences the accurate judgement of distance. Nature, 395:497-500, 1998.

[24] K. Tanaka, J. Hayashi, M. Inami, and S. Tachi. Twister: an immersive autostereoscopic display. In Virtual Reality, 2004. Proceedings. IEEE, pages 59-278, March 2004.

[25] B. G. Witmer and M. J. Singer. Measuring presence in virtual environments: A presence questionnaire. Presence: Teleoper. Virtual Environ., 7(3):225-240, 1998.

[26] B. Wu, T. L. Ooi, and Z. J. He. Perceiving distance accurately by a directional process of integrating ground information. Nature, 428:7377, 2004.

[27] N. Zikic. Evaluating Relative Impact of VR Components Screen size, Stereoscopy and Field of View on Spatial Comprehension and Presence in Architecture. PhD thesis, The Pennsylvania State University, University Park, PA, USA, 2007. 